A binocular test of sensory eye dominance based on the Pulfrich effect

\author{
Nicola Megna, Giampaolo Lucarini, Alessandro Fossetti \\ Istituto di Ricerca e di Studi in Ottica e Optometria, Vinci, Italy
}

\begin{abstract}
Author Note
$5 \quad$ Nicola Megna (iD) https://orcid.org/0000-0002-4552-8074

Correspondence concerning this article should be addressed to Nicola Megna,

Istituto di Ricerca e di Studi in Ottica ed Optometria, Piazza della Libertà 18, 50059 Vinci

(FI), Italy. E-mail: nicolamegna@gmail.com
\end{abstract}




\begin{abstract}
Many studies have shown inconsistent results among traditional measures of sensory ocular dominance (SED), seriously questioning the very concept of SED as a unitary aspect of the visual system (e.g. Mapp et al., 2003, Skottun \& Freeman, 1984, Walls, 1951). The test outcomes may also change even if the same measure is repeated under different conditions, for example by varying the distance and eccentricity of the target (e.g. Khan \& Crawford, 2001; Rice et al., 2008). On the other hand, some authors suggest that such inconsistencies may result from uncontrolled variables. A candidate to produce confusing variables is the frequent format of these tests, often dichotomous and introspective.

In this paper, we propose a possibility of measuring SED on a continuous scale and in a comparative way using a stimulus that induces a Pulfrich effect. Here a dichoptic motion stimulus, borrowed from a previous study (Reynaud \& Hess, 2017), was used, which produced different degrees of 3D illusory perception strength through the variation of retinal disparity. We observed that the responses of the subjects varied according to their classical SED test outcomes and we estimated the differences in terms of the time delay of the information coming from the two eyes. In our sample, it appears that information from the dominant eye was processed $8.2 \pm 5.8 \mathrm{~ms}$ faster than that of the fellow eye. People with a left dominant eye showed more marked differences in processing time $(6.8 \pm 2.0 \mathrm{~ms})$ than people with a right dominant eye $(1.8 \pm 0.9 \mathrm{~ms})$. Eyes without a clear dominance did not show significant differences in processing time $(1.2 \pm 1.7 \mathrm{~ms})$. These results are consistent with the previous literature and could lead to the development of a new continuous-scaled SED test.
\end{abstract}

10

Keywords: eye dominance, Pulfrich effect, psychophysics, methodology 


\section{A binocular test of sensory eye dominance based on the Pulfrich effect}

\section{Background}

Binocular vision is based on an asymmetric use of the two eyes (Ehrenstein et al.,

35

2005: Freeman \& Ohzawa, 1990 Porac \& Coren, 1975; Rakic, 1976, Skottun \& Freeman, 1984) not only in amblyopic subjects (Daw, 1998, Horton \& Hocking, 1996, Schröder et al., 2002 ) but also under physiological conditions (Chaurasia \& Mathur, 1976, Ehrenstein et al., 2005, Reiss \& Reiss, 1997). As for other members of any bilateral pair of structures of the body, such as hands, this kind of functional priority or preferential activity is commonly called dominance. Approximately two-thirds of the population has a dominant right eye and only a small portion seems to have balanced eyes (Chaurasia \& Mathur, 1976 Kommerell et al., 2003). Determining eye dominance (ED) can be important 1) in clinical optometry contexts, e.g. for monovision, cataract and refractive surgery and contact lens wear (Evans, 2007; Handa et al., 2004; Handa et al., 2005: McMonnies, 1974; Zheleznyak et al., 2015), 2) in experimental contexts, e.g. for accurate eye movements measures (Chaumillon et al., 2017), 3) in sports, when one eye is used more than the other, such as shooting, baseball and golf (Dalton et al., 2015; Jones 3rd et al., 1996, Laby et al., 1998). Some studies reported that information from the dominant eye is faster (Metalis \& Niemiec, 1984; Minucci \& Connors, 1964) and more salient (Coren \& Porac, 1976, Pascal, 1926; Porac \& Coren, 1979, 1981; Wade, 1975) than information from the non-dominant eye. Sensory eye dominance (SED) appears to be based on an imbalance of inhibitory connections between the two eyes in various brain areas (Sengpiel et al., 1998; Sengpiel \& Blakemore, 1996 Sengpiel et al., 2006). Notably, changes in cortical metabolism of V1 that depended on SED have been recorded with functional magnetic resonance imaging (fMRI) in the human brain (Rombouts et al., 1996).

Many authors have proposed a surprising number of criteria to determine ED (Gronwall \& Sampson, 1971; Jasper \& Raney, 1937; Lederer, 1961; Walls, 1951), to the point to questioning the usefulness of the very concept of dominance as a unitary 
characteristic of vision (Laby \& Kirschen, 2011; Mapp et al., 2003; Walls, 1951). Two types

60

65

of ED, motor dominance (MD) and sensory dominance, are identified through several clinical tests (Li et al., 2010, Pointer, 2012). Although their satisfactory test-retest repeatability, they are generally inconsistent with each other and depend crucially upon a number of other variable, e.g. the distance to the target and the direction of gaze (Ho et al., 2018; Jasper \& Raney, 1937; Khan \& Crawford, 2001; Mapp et al., 2003; Rice et al., 2008; Walls, 1951). This leads many researchers to question their validity. Since working tests of the same property should give the same result, there must be either a problem with the tests or with the understanding of the ED. Walls (1951) listed 25 different ways to test and conceptualise ED and proposed that only the measurements that involved stereopsis and fusion were actually about dominance. In other terms, tests that do not allow the subjects to use their normal binocular vision may not accurately assess SED. In their review, Laby and Kirschen (2011) confirmed that only tests that allow for maintenance of binocular vision should be used to accurately evaluate SED. On the other hand, it is possible that ED is a multifactorial phenomenon and there could exist several types of dominance (Mapp et al., 2003; Rice et al., 2008).

Although eye dominance and handedness are not related (Mapp et al., 2003; Rice et al., 2008, Walls, 1951), the research on these two topics is much more similar than it may appear at first glance, and bringing them together might produce some insight. Commonly we talk about right-handed and left-handed people, but it is long been known that handedness is not dichotomous. The Edinburgh Inventory (Oldfield, 1971) is a set of items investigating the ways people use their hands in various contexts. In a variable number of these items, people do not report the same preferred hand. Researchers do not interpret these results as a problem with the construct of handedness, but as the evidence that it is not a dichotomous variable: there exist more degrees of handedness that are expressed by the level of coherence of the responses to the test, and the more the coherence, the more the degree of laterality (Isaacs et al., 2006). From the Edinburgh Inventory, it is possible to 
obtain an index, the Laterality Quotient, ranging from -100 (pure left) to 100 (pure right), in which to place the handedness of each person. The underlying logic is that a construct that is not directly observable (for example, SED) may manifest itself in different ways from person to person. If the manifestations (phenomena) do not agree with each other, this does not necessarily mean that the construct is faulty, but that it might be necessary to discuss how the related observations are measured and interpreted.

Back in the field of vision research, we point out that generally SED classification in the clinical practice is made on a dichotomous basis. Indeed, significant efforts to develop continuous scale tests have been made in experimental research (see Bossi et al. (2018) for a review). Another problematic feature of clinical sensory testing is to require subjects to perform an introspective task. For example, when using a red filter or fogging one eye with a positive lens, the observer is asked to report when his perception of a target is most disrupted, and no performance index is recorded. The use of introspection poses various epistemological and methodological problems (Gronwall \& Sampson, 1971).

Bossi et al. (2018) reviewed eight methods for quantifying SED, six of which are based on the individuation of a balance point (BP) or a point of subjective equality (PSE) between the two eyes regarding a particular parameter of the stimulus. For instance, in the Letter-Polarity test, two superimposed letters are presented with opposite luminance polarity to the two eyes, and the observer is asked to report which letter appears brighter: the SED measure, in this case, is the contrast balance point between the two eyes (Bossi et al., 2017), which can be derived from the psychometric function between the proportion of responses in a 2 alternative forced-choice task and the respective stimulus intensities. This method allows a continuous and performance-based measure of SED, but still, the eyes compete with each other rather than cooperate to process the information.

A recent study describes an interesting paradigm that might be used in continuous-scaled and objective ED measurements where eyes do not compete but cooperate to perception with their imbalanced contributions, as recommended by some 
authors (Laby \& Kirschen, 2011; Walls, 1951). In 2017, Reynaud and Hess (2017) used a dichoptic paradigm in which an illusory 3D percept for a moving object generated

The present work aims to replicate the results of Reynaud and Hess (2017) and to investigate the possibility that the innate bias that they discovered consists in the observers' SED. If this is the case, their paradigm could be the basis for a new 

in stereopsis (Laby \& Kirschen, 2011; Walls, 1951).

\section{Methods}

\section{Setup and stimuli}

In order to implement the experiment and analyse data we used an Apple MacBookAir 13" (2017) with a $1.8 \mathrm{GHz}$ Intel Core i5 processor, 8 Gb RAM and an Intel HD Graphics 60001536 MB graphic card. The operating system was MacOs Mojave 10.14.2. The monitor had a 1440x900 pixels resolution with pixel size $0.02 \mathrm{~cm}$ and a $60 \mathrm{~Hz}$ refresh rate and was calibrated using a Minolta LS-100 photometer. The data was collected in a dimly lit room. We used Python 2.7 in conjunction with many modules as for example SciPy (Jones et al., 2001), Numpy (Oliphant, 2006), Matplotlib (Hunter, 2007) and PsychoPy (Garaizar \& Vadillo, 2014; J. Peirce \& MacAskill, 2018; J. W. Peirce, 2007, 2009).

The stimuli presented to the two eyes were fused through two $8 \Delta$ prisms mounted on a stereoscope created ad-hoc by our laboratory, as shown in Figure 1. They consisted of two groups of 100 Gabor patches, each with $\sigma=0.25^{\circ}$, random phase, and spatial frequency $=2.5 \mathrm{c} / \mathrm{deg}$, that were displayed for $1.5 \mathrm{~s}$ independently to the two eyes. The viewing distance was $75 \mathrm{~cm}$. Gabors presented a 100\% coherent harmonic motion that gave to the subject an impression of a $8 \times 6^{\circ}$ cylinder rotating on its axis at $23^{\circ} / \mathrm{s}$. The two cylinders were presented dichotopically to the subject (Figure 1). Two parameters could be manipulated:

1. the angular phase difference between the two stimuli, that could vary randomly on a trial basis from $0.04^{\circ}$ to $0.75^{\circ}$ in log progression. Crucially, the illusory tridimensionality of the rotating cylinder and the direction of rotation depended directly on this parameter: coherently to the Pulfrich effect, when the Gabors on the right sides preceded their correspondent Gabors on the left, subjects perceived a 
clockwise rotation; otherwise, when left Gabors preceded right Gabors, subjects perceived a counter-clockwise rotation (Pulfrich, 1922, Reynaud \& Hess, 2017; Treue et al., 1991).

2. the interocular contrast difference between the two stimuli, that varied on a session basis. The contrast of one cylinder could be decreased by $0 \%, 30 \%$, or $60 \%$ with a mean luminance of $156 \mathrm{~cd} / \mathrm{m}^{2}$. Coherently to the Pulfrich effect (Farr et al., 2018, Pulfrich, 1922, Reynaud \& Hess, 2017), this parameter affected the perceived rotation of the stimulus.

\section{Procedure}

After been provided with a first test session to become familiar with the stimulus and task, subjects collected at least 100 trials per contrast condition. Each session started with the presentation of two red circumferences containing two letters, $F$ and $L$. When the observer reported perceiving the letter $E$, fusion was obtained and the experiment started when the observer pressed any key on the keyboard. After the presentation of the stimulus for $1.5 \mathrm{~s}$, subjects were asked to report the perceived orientation of the cylinder by pressing the left or right arrow on the keyboard. During the test session, the angular phase difference was high to be confident that the subject could understand the task. During the experiment sessions, the angular phase difference randomly varied on a trial basis while the contrast of the left or the right stimulus varied on a session basis.

The ED of each subject was assessed based on three traditional tests, one for motor dominance (pointing test) and two for sensorial dominance (Bagolini's red filter and 1.5 $\Delta$ positive lens tests), with a target stimulus distance of $4 \mathrm{~m}$. We decided to use these tests and conditions because of their widespread use in the professional community. Observers were classified as showing an uncertain dominance (ND) when their introspective response was still uncertain after the second measurement attempt. 


\section{Statistical analysis}

The ED of each observer was classified based on the traditional testing results. When the three tests were consistent, the observer was assigned to the right (RE) or left (LE) dominance groups. When at least one test was inconsistent with the others or resulted uncertainly, the observer's dominance was classified as null (ND). Similar methods of classification have been already used in other recent studies (e.g. Lopes-Ferreira et al., 2013).

As in Reynaud and Hess (2017), we calculated the point of subjective equality (PSE) in terms of angular phase difference from the psychometric curves that we obtain for each contrast condition. The PSE indicates the angular phase difference at which the subject could not tell the motion orientation of the cylinder. We fitted the data with a logistic curve:

$$
f(x)=\frac{a}{1+b \cdot e^{-c \cdot x}}+d
$$

where $a$ indicates the superior asymptote of the curve, $b$ and $d$ are the positions of the curve relative to the $\mathrm{x}$-axis and the $\mathrm{y}$-axis respectively, and $c$ is the slope. Since we wanted to force (1) to be limited from 0 to 1 along the y-axis, we used the equation:

$$
f(x)=\frac{1}{1+b \cdot e^{-c \cdot x}}
$$

The PSE corresponds to the inflection point for $(2)$ when the $f(x)=0.5$, that is:

$$
P_{x_{(P S E)}, y_{(0.5)}}=\left(\frac{\ln b}{c}, 0.5\right)
$$

The parameters $b$ and $c$ were derived from the logistic curve that best fitted the data points using a least-square estimation.

The normality of the sample PSE distributions for each dominance category and the equality of their variances were assessed by performing a Shapiro-Wilk test (Kleinbaum et al., 2013 Shapiro \& Wilk, 1965) and a Fisher F test (Box, 1953), respectively. We performed an ANOVA followed by two-tailed t-tests to compare the mean PSEs for 
different EDs when the Shapiro-Wilk and F tests indicated that the assumptions for an ANOVA were respected (Scheffé, 1953). In case it was not possible to perform an ANOVA,

we made the comparisons using a t-test with a Bonferroni correction (Abdi et al., 2007;

Bonferroni, 1936). Finally, in order to decide if the observed consistencies between the traditional ED tests were significant, we performed a one-tailed binomial test on their successfully coherent output rate (Dodge, 2008).

We performed a sample size estimation (Cohen, 2013) by using the data recollected by Reynaud and Hess (2017) to decide in advance the number of subjects to be enrolled in this study to have sufficient statistical power to detect a difference between the PSE as a function of ocular dominance.

\section{Participants}

Nineteen observers (53\% men, $47 \%$ women; age $=26 \pm 8$ ) participated in the experiment. All of them did not present any pathological abnormality and had a visual acuity of $6 / 6$ or better when corrected. Two out of them could not fuse the stimuli and other four participants were excluded because their responses were invariant. Indeed, they perceived the stimulus as rotating in the same direction $>85 \%$ of times, and no psychometric curve could be obtained from the data. Our tentative interpretations for this behavior are illustrated in the Discussion section.

\section{Results}

\section{Descriptive statistics and traditional test results}

Table 1 reports the results in the traditional dominance tests. In the pointing test, $41 \%$ of observers showed a right dominance, and $53 \%$ showed a left dominance. One observer $(6 \%)$ could not perform the task nor fuse together the targets. In the red filter test, $24 \%$ of observers showed a right dominance, $35 \%$ showed a left dominance and $41 \%$ did not show a clear dominance. In the positive lens test, $35 \%$ of observers showed a right 
dominance, $47 \%$ a left dominance, and $18 \%$ showed an uncertain dominance. Comparing the outputs of each test on a within-subject basis, we observed that the most consistent observers were consistently classified as left-dominants (LD).

\section{Psychometric curves and PSE estimations}

Figures 2a, 2b and 2c show the individual psychometric curves in five interocular contrast difference conditions for three observers. The curves express the relation between the perceived rotation and the angular phase difference between eyes. When this difference was $-0.75^{\circ}$, observers perceive counter-clockwise motion and when it was $0.75^{\circ}$, they perceive a clockwise motion. These results are consistent with the well-known Pulfrich effect and are dependent on the interocular delay of visual information (Anzai et al., 2001; Pulfrich, 1922 Reynaud \& Hess, 2017). Interestingly, reversals of the perceived rotation observers, the PSE values were negative as in the case of FN (Figure 2a and 2d). Notably, their PSEs did remain negative even when the contrast of the right stimulus was decreased, as observed by Reynaud and Hess (2017). Other observers presented the opposite pattern with positive PSEs, as in the case of FP (Figure 2c and 2f) and a few participants did show near-zero PSEs with an inversion of their sign when interocular contrast difference varied (Figure 2b and 2e). 


\section{Comparison between traditional tests and PSE estimations}

The results presented in the previous section confirm the observations of Reynaud and Hess (2017) and, in particular, we found evidence for what they called "an innate bias"

The histograms reported in Figures 3d 3f refer to the mean PSEs grouped only for the pointing, red filter or positive lens tests. The mean PSEs of left motor dominance 
subjects $(\mathrm{N}=6)$ do not differ from those of right motor dominance subjects $(\mathrm{N}=7)$ (figure

\section{Discussion}

After replicating the results of Reynaud and Hess (2017), a substantial difference was observed between the PSEs of people with left and right eye dominance, which can be easily converted into the temporal domain in terms of interocular delay (ID). Since the stimulus presented a rotation of about $23^{\circ} / \mathrm{s}$, our results indicate that information from the dominant eye could anticipate information from the fellow eye of about $8.2 \pm 5.8 \mathrm{~ms}$. Notably, while balanced eyes seem to have a non-significant temporal difference (ID=1.2 $\pm 1.7 \mathrm{~ms}$ ), left dominant eyes show a more pronounced temporal difference (ID=6.8 $\pm 2.0 \mathrm{~ms})$ than right dominant eyes ( $\mathrm{ID}=1.5 \pm 0.9 \mathrm{~ms})$.

A possible explanation of the asymmetry observed in this study might derive from a 
315 temporal delay between the two eyes, and when this delay exceeds the integration time the 
inputs coming from the two eyes become completely unrelated. The amplitude of this temporal window for the retinal disparity is $16 \pm 5 \mathrm{~ms}$, a value that is consistent with our results. Interestingly, Read and Cumming (2005a) have observed, similarly to what we stereoscopic vision in the norm), for $20 \%$ of the subjects we had to proceed to their exclusion because they had a rigid response set that prevented the measurement of a psychometric curve. At the moment we can only explain this assuming that these subjects 
tended to respond based on a certain perceptual rigidity or influenced by an attentive bias subject's introspection, which can be a useful tool to quantify the extent of eye dominance that, with the appropriate modifications, could be used by clinicians and experimenters to quantify the sensory eye dominance. 
Abdi, H. et al. (2007). Bonferroni and šidák corrections for multiple comparisons. Encyclopedia of measurement and statistics, 3, 103-107.

Anzai, A., Ohzawa, I., \& Freeman, R. D. (2001). Joint-encoding of motion and depth by visual cortical neurons: Neural basis of the pulfrich effect. Nature neuroscience, $4(5), 513-518$.

Blake, R., \& Logothetis, N. K. (2002). Visual competition. Nature Reviews Neuroscience, $3(1), 13-21$.

Bonferroni, C. (1936). Teoria statistica delle classi e calcolo delle probabilita. Pubblicazioni del $R$ Istituto Superiore di Scienze Economiche e Commericiali di Firenze, 8, 3-62.

Bossi, M., Hamm, L. M., Dahlmann-Noor, A., \& Dakin, S. C. (2018). A comparison of tests for quantifying sensory eye dominance. Vision research, 153, 60-69.

Bossi, M., Tailor, V. K., Anderson, E. J., Bex, P. J., Greenwood, J. A., Dahlmann-Noor, A., \& Dakin, S. C. (2017). Binocular therapy for childhood amblyopia improves vision without breaking interocular suppression. Investigative ophthalmology \& visual science, 58(7), 3031-3043.

Box, G. E. (1953). Non-normality and tests on variances. Biometrika, 40(3/4), 318-335.

Bradley, D. C., Qian, N., \& Andersen, R. A. (1995). Integration of motion and stereopsis in middle temporal cortical area of macaques. Nature, 373(6515), 609-611.

Burr, D. C., \& Ross, J. (1979). How does binocular delay give information about depth? Vision research, $19(5), 523-532$.

Caparos, S., Fortier-St-Pierre, S., Gosselin, J., Blanchette, I., \& Brisson, B. (2015). The tree to the left, the forest to the right: Political attitude and perceptual bias. Cognition, 134, 155-164.

Chaumillon, R., Alahyane, N., Senot, P., Vergne, J., Lemoine-Lardennois, C., Blouin, J., Doré-Mazars, K., Guillaume, A., \& Vergilino-Perez, D. (2017). Asymmetry in visual 
information processing depends on the strength of eye dominance. Neuropsychologia, $96,129-136$.

Chaurasia, B., \& Mathur, B. (1976). Eyedness. Cells Tissues Organs, 96(2), 301-305.

Cohen, J. (2013). Statistical power analysis for the behavioral sciences. Academic press.

Farr, J., McGarva, E., Nij Bijvank, J., van Vliet, H., Jellema, H. M., Crossland, M. D., \& Petzold, A. (2018). The pulfrich phenomenon: Practical implications of the assessment of cases and effectiveness of treatment. Neuro-ophthalmology, 42(6), $349-355$.

440

Freeman, R. D., \& Ohzawa, I. (1990). On the neurophysiological organization of binocular vision. Vision research, 30(11), 1661-1676. 
Garaizar, P., \& Vadillo, M. A. (2014). Accuracy and precision of visual stimulus timing in psychopy: No timing errors in standard usage. PloS one, 9(11), e112033.

Geisler, W. S., Albrecht, D. G., \& Crane, A. M. (2007). Responses of neurons in primary visual cortex to transient changes in local contrast and luminance. Journal of Neuroscience, 27(19), 5063-5067.

Gronwall, D., \& Sampson, H. (1971). Ocular dominance: A test of two hypotheses. British Journal of Psychology, 62(2), 175-185.

Handa, T., Mukuno, K., Uozato, H., Niida, T., Shoji, N., Minei, R., Nitta, M., \& Shimizu, K. (2004). Ocular dominance and patient satisfaction after monovision induced by intraocular lens implantation. Journal of Cataract $\&$ Refractive Surgery, $30(4), 769-774$.

Handa, T., Shimizu, K., Mukuno, K., Kawamorita, T., \& Uozato, H. (2005). Effects of ocular dominance on binocular summation after monocular reading adds. Journal of Cataract \& Refractive Surgery, 31 (8), 1588-1592.

Heravian-Shandiz, J., Douthwaite, W., \& Jenkins, T. (1991). Binocular interaction with neutral density filters as measured by the visual evoked response. Optometry and vision science: official publication of the American Academy of Optometry, 68(10), $801-806$.

Ho, R., Thompson, B., Babu, R. J., \& Dalton, K. (2018). Sighting ocular dominance magnitude varies with test distance. Clinical and Experimental Optometry, $101(2)$, 276-280.

Hogeboom, M., \& van Leeuwen, C. (1997). Visual search strategy and perceptual organization covary with individual preference and structural complexity. Acta Psychologica, 95(2), 141-164.

Horton, J. C., \& Hocking, D. R. (1996). Pattern of ocular dominance columns in human striate cortex in strabismic amblyopia. Visual neuroscience, 13(4), 787-795. 
Hunter, J. D. (2007). Matplotlib: A 2d graphics environment. IEEE Annals of the History of Computing, 9(03), 90-95.

Isaacs, K. L., Barr, W. B., Nelson, P. K., \& Devinsky, O. (2006). Degree of handedness and cerebral dominance. Neurology, 66(12), 1855-1858.

Jasper, H. H., \& Raney, E. T. (1937). The phi test of lateral dominance. The American Journal of Psychology, 49(3), 450-457.

Jones, E., Oliphant, T., Peterson, P., et al. (2001). Scipy: Open source scientific tools for python.

Jones 3rd, L., Classe, J. G., Hester, M., \& Harris, K. (1996). Association between eye dominance and training for rifle marksmanship: A pilot study. Journal of the American Optometric Association, 67(2), 73-76.

Katsumi, O., Tanino, T., \& Hirose, T. (1986). Objective evaluation of binocular function using the pattern reversal visual evoked response. ii. effect of mean luminosity. Acta ophthalmologica, 64 (2), 199-205.

Khan, A. Z., \& Crawford, J. D. (2001). Ocular dominance reverses as a function of horizontal gaze angle. Vision research, 41(14), 1743-1748.

Kleinbaum, D. G., Kupper, L. L., Nizam, A., \& Rosenberg, E. S. (2013). Applied regression analysis and other multivariable methods. Cengage Learning.

Kommerell, G., Schmitt, C., Kromeier, M., \& Bach, M. (2003). Ocular prevalence versus ocular dominance. Vision Research, 43(12), 1397-1403.

Laby, D. M., \& Kirschen, D. G. (2011). Thoughts on ocular dominance - is it actually a preference? Eye $\&$ contact lens, 37(3), 140-144.

Laby, D. M., Kirschen, D. G., Rosenbaum, A. L., \& Mellman, M. F. (1998). The effect of ocular dominance on the performance of professional baseball players. Ophthalmology, $105(5), 864-866$.

Lages, M., Mamassian, P., \& Graf, E. W. (2003). Spatial and temporal tuning of motion in depth. Vision Research, 43(27), 2861-2873. 
Lederer, J. (1961). Ocular dominance. Clinical and Experimental Optometry, 44(11), $531-574$

Li, J., Lam, C. S., Yu, M., Hess, R. F., Chan, L. Y., Maehara, G., Woo, G. C., \& Thompson, B. (2010). Quantifying sensory eye dominance in the normal visual system: A new technique and insights into variation across traditional tests. Investigative Ophthalmology $\&$ Visual Science, 51(12), 6875-6881.

Lopes-Ferreira, D., Neves, H., Queiros, A., Faria-Ribeiro, M., Peixoto-de-Matos, S., \& González-Méijome, J. M. (2013). Ocular dominance and visual function testing. BioMed research international, 2013.

Mapp, A. P., Ono, H., \& Barbeito, R. (2003). What does the dominant eye dominate? a brief and somewhat contentious review. Perception \& psychophysics, 65(2), 310-317.

McMonnies, C. W. (1974). Monocular fogging in contact lens practice. The Australian Journal of Optometry, 57(1), 28-32.

Metalis, S. A., \& Niemiec, A. J. (1984). Assessment of eye dominance through response time. Perceptual and motor skills, 59(2), 539-544.

Minucci, P. K., \& Connors, M. M. (1964). Reaction time under three viewing conditions: Binocular, dominant eye, and nondominant eye. Journal of Experimental psychology, 67(3), 268.

Oldfield, R. C. (1971). The assessment and analysis of handedness: The edinburgh inventory. Neuropsychologia, 9(1), 97-113.

Oliphant, T. E. (2006). A guide to numpy (Vol. 1). Trelgol Publishing USA.

Pascal, J. I. (1926). The chromatic test for the dominant eye. Am J Ophthalmol, 9, 357-358.

Peirce, J., \& MacAskill, M. (2018). Building experiments in psychopy. Sage.

Peirce, J. W. (2007). Psychopy-psychophysics software in python. Journal of neuroscience methods, 162(1-2), 8-13.

Peirce, J. W. (2009). Generating stimuli for neuroscience using psychopy. Frontiers in neuroinformatics, 2, 10. 
Pointer, J. S. (2012). Sighting versus sensory ocular dominance. Journal of Optometry, $5(2), 52-55$.

Porac, C., \& Coren, S. (1975). Suppressive processes in binocular vision: Ocular dominance and amblyopia. American journal of optometry and physiological optics, 52(10), $651-657$.

Porac, C., \& Coren, S. (1979). Monocular asymmetries in recognition after an eye movement: Sighting dominance and dextrality. Perception \& Psychophysics, 25(1), $55-59$.

Porac, C., \& Coren, S. (1981). Lateral preferences and human behavior. Springer.

Pulfrich, C. (1922). Die stereoskopie im dienste der isochromen und heterochromen photometrie. Naturwissenschaften, 10(35), 751-761.

Rakic, P. (1976). Prenatal genesis of connections subserving ocular dominance in the rhesus monkey. Nature, 261(5560), 467-471.

Raz, D., Seeliger, M. W., Geva, A. B., Percicot, C. L., Lambrou, G. N., \& Ofri, R. (2002). The effect of contrast and luminance on mferg responses in a monkey model of glaucoma. Investigative ophthalmology \& visual science, 43(6), 2027-2035.

Read, J. C., \& Cumming, B. G. (2005a). Effect of interocular delay on disparity-selective v1 neurons: Relationship to stereoacuity and the pulfrich effect. Journal of Neurophysiology, 94(2), 1541-1553.

Read, J. C., \& Cumming, B. G. (2005b). The stroboscopic pulfrich effect is not evidence for the joint encoding of motion and depth. Journal of Vision, 5(5), 3-3.

Reiss, M., \& Reiss, G. (1997). Ocular dominance: Some family data. Laterality: Asymmetries of Body, Brain and Cognition, 2(1), 7-16.

Reynaud, A., \& Hess, R. F. (2017). Interocular contrast difference drives illusory 3d percept. Scientific reports, 7(1), 1-6. 
Rice, M. L., Leske, D. A., Smestad, C. E., \& Holmes, J. M. (2008). Results of ocular dominance testing depend on assessment method. Journal of American Association for Pediatric Ophthalmology and Strabismus, 12(4), 365-369.

Rombouts, S. A., Barkhof, F., Sprenger, M., Valk, J., \& Scheltens, P. (1996). The functional basis of ocular dominance: Functional mri (fmri) findings. Neuroscience Letters, 221 (1), 1-4.

Sanada, T. M., \& DeAngelis, G. C. (2014). Neural representation of motion-in-depth in area mt. Journal of Neuroscience, $34(47), 15508-15521$.

Scheffé, H. (1953). A method for judging all contrasts in the analysis of variance. Biometrika, 40(1-2), 87-110.

Schröder, J.-H., Fries, P., Roelfsema, P. R., Singer, W., \& Engel, A. K. (2002). Ocular dominance in extrastriate cortex of strabismic amblyopic cats. Vision research, 42(1), 29-39.

Sengpiel, F., Baddeley, R. J., Freeman, T. C., Harrad, R., \& Blakemore, C. (1998). Different mechanisms underlie three inhibitory phenomena in cat area 17. Vision research, 38(14), 2067-2080.

Sengpiel, F., \& Blakemore, C. (1996). The neural basis of suppression and amblyopia in strabismus. Eye, 10(2), 250-258.

Sengpiel, F., Jirmann, K.-U., Vorobyov, V., \& Eysel, U. T. (2006). Strabismic suppression is mediated by inhibitory interactions in the primary visual cortex. Cerebral cortex, $16(12), 1750-1758$.

Shapiro, S. S., \& Wilk, M. B. (1965). An analysis of variance test for normality (complete samples). Biometrika, 52(3/4), 591-611.

Skottun, B., \& Freeman, R. (1984). Stimulus specificity of binocular cells in the cat's visual cortex: Ocular dominance and the matching of left and right eyes. Experimental Brain Research, 56(2), 206-216. 
Tchanturia, K., Serpell, L., Troop, N., \& Treasure, J. (2001). Perceptual illusions in eating disorders: Rigid and fluctuating styles. Journal of behavior therapy and experimental psychiatry, 32(3), 107-115.

Treue, S., Husain, M., \& Andersen, R. A. (1991). Human perception of structure from motion. Vision research, 31(1), 59-75.

Wade, N. (1975). Fragmentation of monocular afterimages in individuals with and without normal binocular vision. Perception $\& 3$ Psychophysics, 18(5), 328-330.

Walls, G. L. (1951). A theory of ocular dominance. AMA archives of ophthalmology, 45(4), $387-412$.

Zheleznyak, L., Alarcon, A., Dieter, K. C., Tadin, D., \& Yoon, G. (2015). The role of sensory ocular dominance on through-focus visual performance in monovision presbyopia corrections. Journal of vision, 15(6), 17-17. 


\section{Table 1}

On the right, $\%$ of observers showing a right, left, or uncertain dominance $(N=17)$. On the left, $\%$ coherence between tests along with Binomial test p-values ( $\alpha=0.016$ after Bonferroni correction)

\begin{tabular}{|l|c|c|c|c|c|c|}
\hline & RE & ND & LE & Pointing & Red filter & Positive lens \\
\hline Pointing & $41 \%$ & $6 \%$ & $53 \%$ & - & - & - \\
Red filter & $24 \%$ & $41 \%$ & $35 \%$ & $71 \%_{(p=0.07)}$ & - & - \\
Positive lens & $35 \%$ & $18 \%$ & $47 \%$ & $76 \%_{(p=0.02)}$ & $71 \%_{(p=0.07)}$ & - \\
\hline
\end{tabular}




\section{Table 2}

Mean PSE values for each dominance groups when merging the results at each test and considering left or right dominant only those who give coherent left or right outcomes (first column), or considering only the result to pointing (second column), red filter (third column) or positive lens (fourth column).

\begin{tabular}{|c|c|c|c|c|}
\hline & Merged & Pointing & Red & Positive \\
\hline RE & $-0.036 \pm 0.041^{\circ}$ & $0.012 \pm 0.086^{\circ}$ & $-0.036 \pm 0.041^{\circ}$ & $-0.017 \pm 0.054^{\circ}$ \\
ND & $0.029 \pm 0.087^{\circ}$ & - & $0.037 \pm 0.098^{\circ}$ & $-0.024 \pm 0.048^{\circ}$ \\
LE & $0.156 \pm 0.094^{\circ}$ & $0.079 \pm 0.12^{\circ}$ & $0.124 \pm 0.1^{\circ}$ & $0.157 \pm 0.081^{\circ}$ \\
\hline
\end{tabular}




\section{Figure 1}

Stimuli were independently presented to the right and left eye through a prismatic lens system that permitted a stereoscopic view. Two $8 \Delta$ prisms were temple mounted on a stereoscope to produce a virtual 3D stimulus. A separating septum made sure that only one of the stimuli on the screen was present on each retina. In the figure, the two black circles represent the eyes and the triangles represent the prisms. The stimuli are represented by a grey rectangle (the monitor) and the virtual stimulus is represented by the dashed rectangle. The stimulus consisted of 200 Gabor patches harmonically moving and presented dichotopically with different angular phase differences and contrast between the two Gabor groups. When the angular phase difference was positive (right>left) subjects tended to perceive a clockwise rotation of the cylinder; when it was negative (right<left), subjects tended to perceive a counter-clockwise rotation. The rotation perception was ambiguous when the angular phase difference was near zero. Interestingly, for many subjects, this zero point in perception did not correspond to the zero point in stimulus intensity, as observed by Reynaud and Hess (2017).

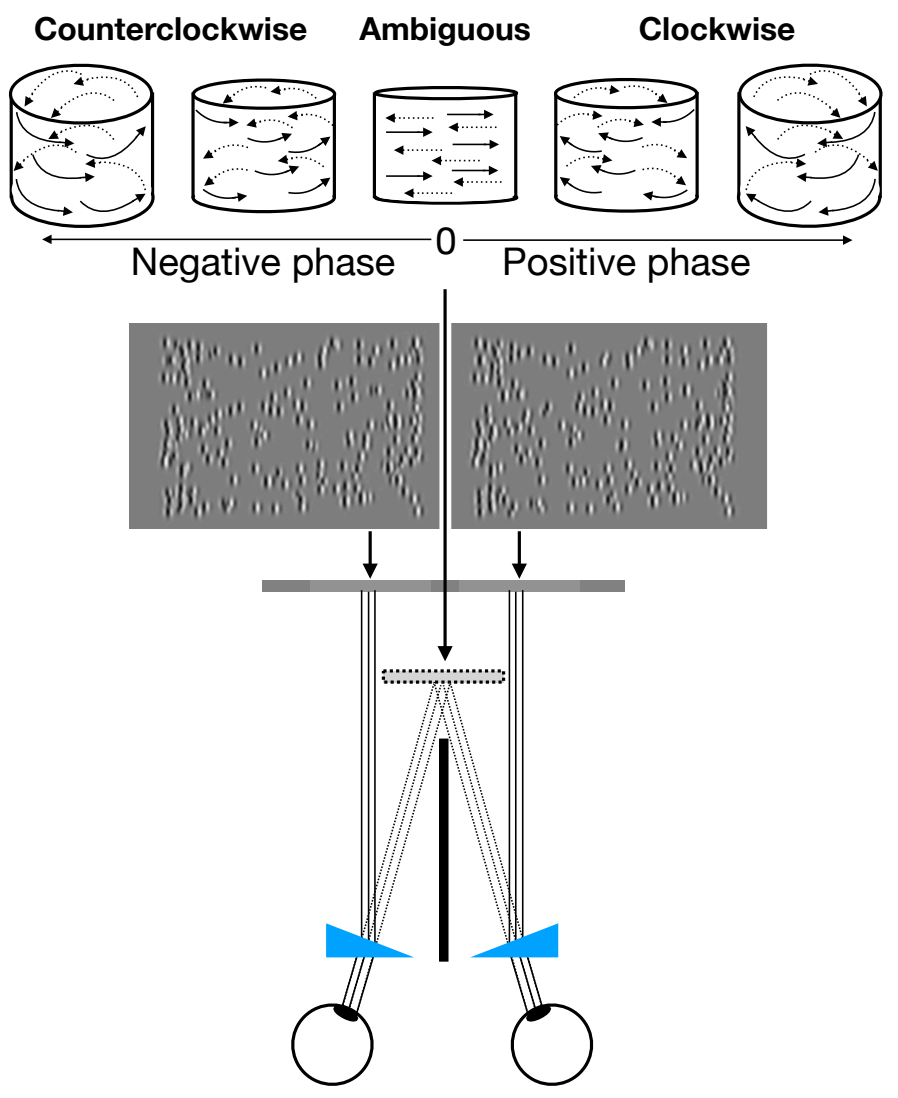




\section{Figure 2}

$\boldsymbol{a}$-c. Individual psychometric functions of the perceived rotation direction for three subjects as a function of the angular phase difference between the stimuli independently presented to the two eyes and as a function of interocular contrast difference. Negative values reported in legends denote a decreased contrast in the left stimulus, positive values denote a decreased contrast in the right stimulus. Data points are fitted by logistic functions from which PSEs are derived (see section 2.4). d-f. PSE values as a function of interocular contrast difference. For most of the subjects a linear function is able to describe this relationship (slopes=[0.13(d), 0.14(e), 0.14(f)]; $r=[0.84(d), 0.93(e), 0.97(f)] ; p=[0.08(d), 0.02(e), 0.005(f)])$. The slopes found in this study are consistent with those reported by Reynaud and Hess (2017)

(a)

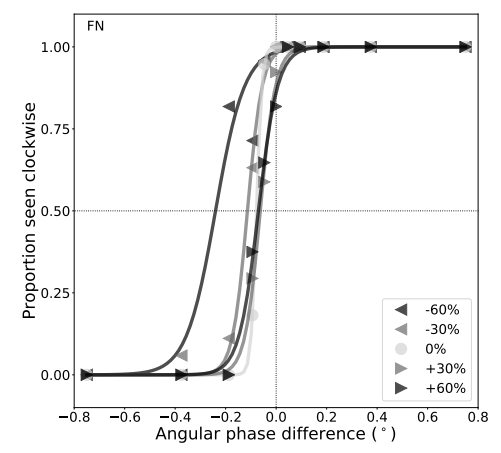

(d)

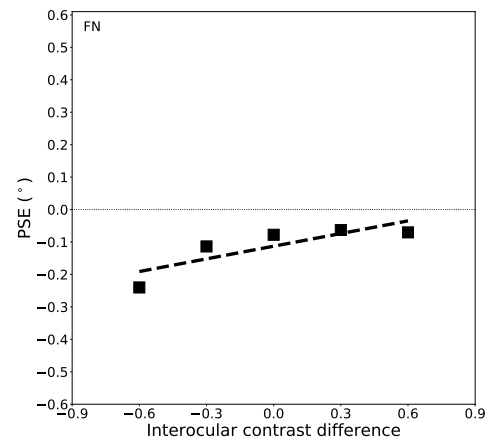

(b)

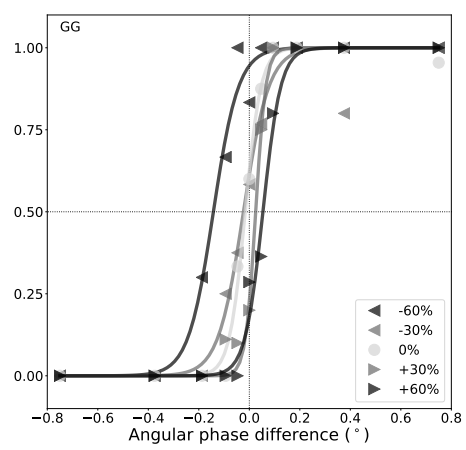

(e)

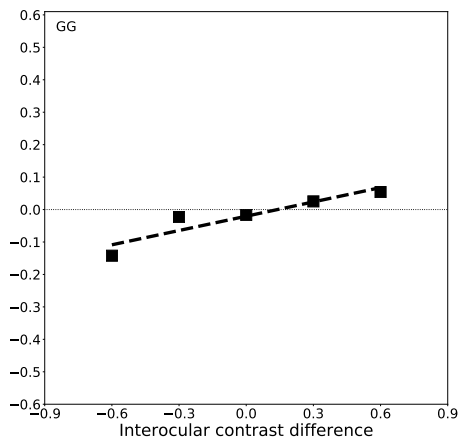

(c)

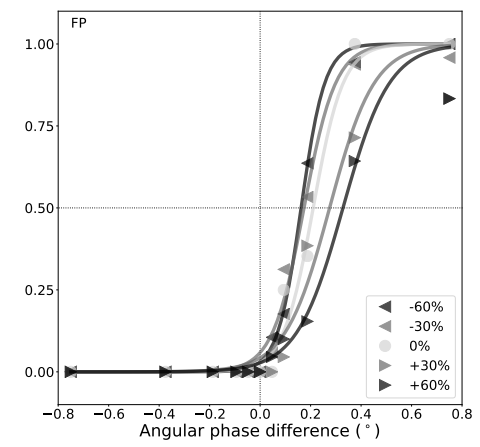

(f)

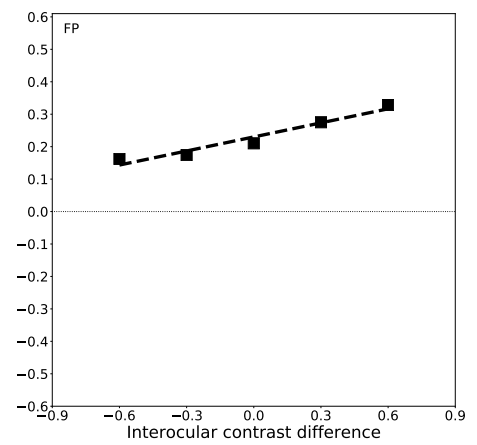




\section{Figure 3}

Mean psychometric curves depicting the proportion of clock-wise perception as a function of angular phase difference for right (RE), left (LE) and uncertain eye dominant (ND) observers as resulted from merging the results of all the traditional tests used (a) and PSEs as a function of interocular contrast difference for the same groups(b). For the three groups, a linear function describes the relation between PSEs and interocular contrast difference (slopes=0.11[RE], $0.11[N D], 0.16[L E] ; r=0.85[R E], 0.87[N D], 0.88[L E] ; p=0.05[R E, N D$ and $L E])$. The slopes found in this study are very close to those reported by Reynaud and Hess (2017), which were about $0.11 \pm 0.07^{\circ}$ (Reynaud $\&$ Hess, 2017). c-f. Histograms of mean PSEs for RE, ND, and LE using four different criteria: merged tests, or using only pointing, red filter, or positive lens test. Each value and significance value is reported in table 2 and the main text.

(a)

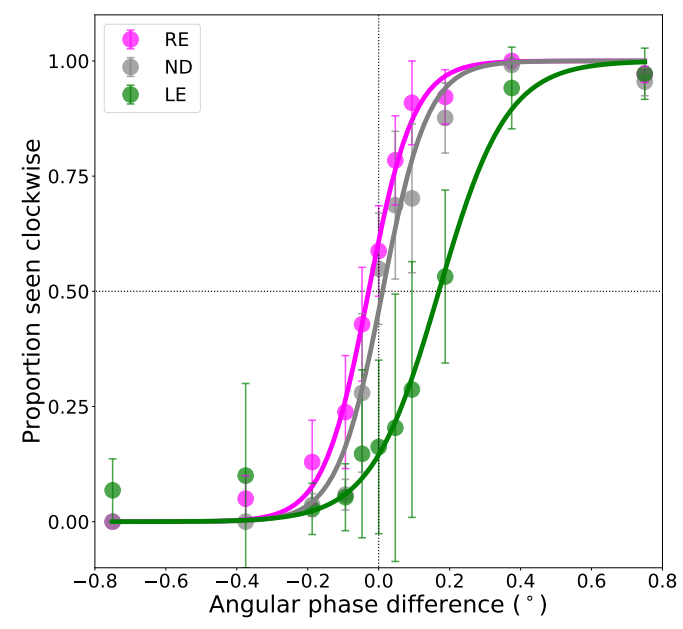

(c) Merged

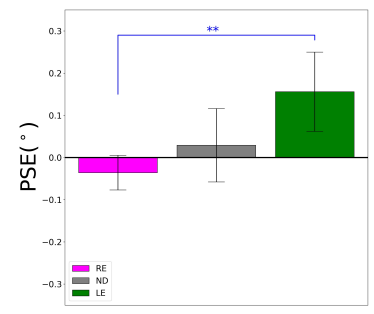

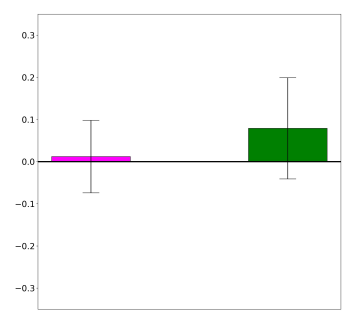

(d) Pointing (b)

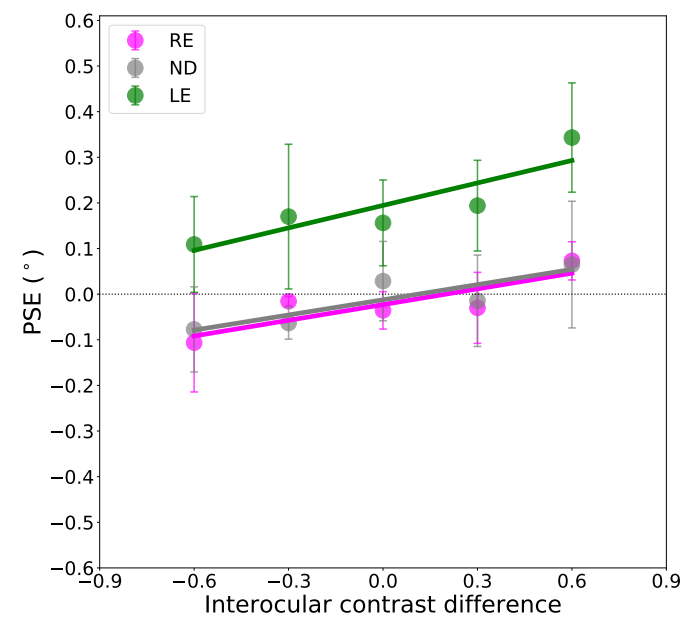

(e) Red

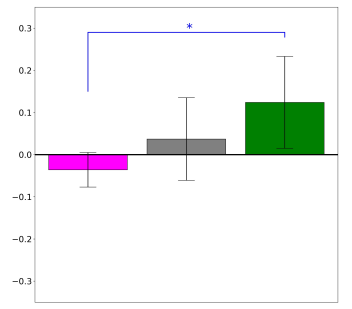

(f) Positive

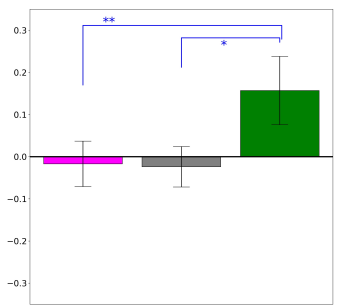

